\title{
Lattices of effectively nonintegral dimensionality
}

\author{
Deepak Dhar* \\ George W. Downs Laboratory of Physics, California Institute of Technology, Pasadena, California 91125 \\ (Received 2 September 1976) \\ We construct a class of lattice systems that have effectively nonintegral dimensionality. A reasonable \\ definition of effective dimensionality applicable to lattice systems is proposed and the effective \\ dimensionalities of these lattices are determined. The renormalization procedure is used to determine the \\ critical behavior of the classical $X Y$ model and the Fortuin-Kasteleyn cluster model on the truncated \\ tetrahedron lattice which is shown to have the effective dimensionality $2 \log 3 / \log 5$. It is found that no \\ phase transition occurs at any finite temperature.
}

\section{INTRODUCTION}

In a recent paper, ${ }^{1}$ Nelson and Fisher defined the truncated tetrahedron lattice. The lattice is planar, multiply connected, and has a coordination number 3 . Although admittedly rather artificial, it is of interest in the theory of phase transitions because it provides us with a lattice of effectively nonintegral dimensionality. Besides providing a physical basis and testing grounds for such formal techniques as the $\epsilon$ expansion, ${ }^{2}$ the study of critical behavior of model Hamiltonians on such lattices may be expected to improve our understanding of the influence of dimensionality in determining the nature of phase transitions in physical systems.

The plan of this paper is as follows: In Sec. I we define what we mean by effective nonintegral space dimensionality of an infinite lattice. The proposed definition is different from that assumed in Ref $\mathrm{f}_{\mathrm{a}}$. In particular the space dimensionality of the truncated tetrahedron lattice using this definition is found to be $2 \ln _{5} 3 \approx 1.3651$ and not $\ln _{2} 3 \approx 1.5851$ as proposed by Nelson and Fisher. Arguments are presented in favor of our definition. In Sec. II two examples of lattice systems with nonintegral dimensionality are given. One is the generalization of the truncated tetrahedron lattice to the truncated $n$-simplex lattice. The other is a planar multiply connected lattice of coordination number 3 which may be obtained by deleting some bonds from a planar square lattice. The dimensionality of these lattices is determined in $\mathrm{Sec}$. III by deriving a functional equation for their characteristic functions. In Sec. IV the behavior of the classical XY model and the cluster model on the truncated tetrahedron lattice is analyzed using the renormalization procedure.

\section{DEFINITION OF THE SPACE DIMENSIONALITY OF AN INFINITE LATTICE}

Consider an infinite lattice. For our purpose a lattice is specified by its graph consisting of lattice points and undirected lines joining them called bonds. For simplicity we consider only one kind of bond. Two sites that have a bond in common are called nearest neighbors. How do we assign a dimensionality to an arbitrary infinite lattice? (The effective dimensionality of a finite lattice may be defined to be zero.) Any proposed definition of effective dimensionality should satisfy some elementary properties. It should agree with the conventional integral value of dimensionality for "regular" lattices. It should depend only weakly on the lattice in the sense that introduction or deletion of a finite number of lattice points or bonds should not change its value. And preferably it should satisfy some scaling relations between critical exponents. ${ }^{3}$

It is instructive to look at the familiar case when the space dimensionality $d$ is integral. We consider a "simple cubic" lattice in $d$ dimensions and consider a model in which a scalar displacement $X_{i}$ is associated with each site $i$ and nearest neighbors are connected by harmonic springs of equal spring constants. Introduction of normal mode coordinates converts this problem into one of independent simple harmonic oscillators. Each normal mode is characterized by a wavenumber $\kappa$ which is a $d$-dimensional vector lying within the first Brillouin zone. We also know that:

(i) For small wavenumbers $\kappa$, the frequency $\omega$ of the mode with wave vector $\kappa$, is approximately proportional to the magnitude of $\kappa$, i.e., $\omega^{2}=\kappa^{2} C^{2}$ for small $\kappa^{2}$.

(ii) The number of modes with $|\kappa|<\kappa$ is proportional to $\kappa^{d}$ for small $\kappa$. The two facts together imply that the fractional number of modes with frequency less than $\omega$ is proportional to $\omega^{d}$ for small $\omega$.

In making a transition to more general lattices with possibly nonintegral dimensionality, we may again define a nearest neighbor harmonic interaction model. It is difficult to say just what meaning may be assigned to a "nonintegral dimensional vector $\kappa$ ". At best we may say that low values of $\mid \boldsymbol{k}$ ! correspond to slowly space varying modes and high values of $|\boldsymbol{k}|$ correspond to the modes where the spatial variation is large. We necessarily assume that something like (ii) is correct. Compare this, for instance, with the scaling property assumed by Wilson [Eqs。(A3) and (A9) in Ref。4]。The validity of (i) is rendered plausible by the observation that the equation $\omega^{2}=\kappa^{2} C^{2}$ is just the Fourier transform of the equation $\partial^{2} \chi / \partial t^{2}=C^{2} \nabla^{2} \chi$. If we identify $\chi_{i}$ as the displacement at site $i$ and replace $\nabla^{2} \chi$ by its discrete analog $\nabla^{2} \chi_{i} \approx \sum_{j}\left(\chi_{j}-\chi_{i}\right)$ where $j$ are sites neighboring $i$, we see that the equation correctly gives the equation of motion of $\chi_{i}$ in our model.

It is thus reasonable to postulate that the fractional number of modes of frequency less than $\omega$ is proportional to $\omega^{d}$, for small $\omega$, even if $d$ is nonintegral. We adopt this as the definition of effective dimensionality of a given infinite lattice.

In practice, the determination of the fractional num- 
ber of modes below a frequency $\omega$ involves starting with a finite lattice of size $N$ and then letting $N$ tend to infinity. For an arbitrary lattice such a limit obviously does not exist. In statistical mechanics, we shall be only concerned with lattices which are sufficiently regular and all such thermodynamic limits will be assumed to exist.

The analysis given in Sec. III shows that the effective dimensionality of the truncated tetrahaedron lattice is $2 \log _{5} 3$.

Nelson and Fisher gave a different argument to determine the effective dimensionality of the truncated tetrahedron lattice. They define the diameter of the lattice as the maximum distance between any two lattice points as measured along bonds. Then it is easy to see that the diameter $R$ of a $r$ th order truncated tetrahedron is proportional to $2^{r}$, while its volume $V$, which is equal to the total number of lattice points, goes like $3^{r}$. Identifying the effective dimensionality $d$ by the relation $V \propto R^{d}$, one finds that $d=\log _{2} 3$ 。

The two definitions of effective dimensionality are clearly not equivalent. The important difference is that while in Nelson and Fisher's definition, one considers the lattice as a whole, our proposed definition looks only at the low spatial frequency modes. Since the phase transitions are governed by the long range correlations, $i_{0} e_{\circ}$, low frequency modes, we believe that our proposed definition is more relevant to the study of phase transitions.

For example, the modified rectangular lattice, described in the next section, has an effective dimensionality $\frac{3}{2}$ using our definition. If we used Nelson and Fisher's definition, the effective dimensionality of the lattice would be 2 . However the behavior of model Hamiltonians like the Gaussian model or the Ising model on this lattice is very different from that on a two-dimensional square lattice. In particular, it may be shown that on this lattice, the Ising model shows no spontaneous magnetization or phase transition. Thus we may expect the assigned effective dimensionality to be less than 2. Similar arguments may be made for the truncated $n$-simplex lattice.

We remark that the effective dimensionality of a lattice has been defined with respect to a specific model (nearest neighbor harmonic spring interaction model). We expect the dimensionality of the lattice to be model independent and different definitions of effective dimensionality using other model Hamiltonians should give an identical value of dimensionality when it is calculated

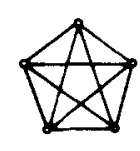

(a)

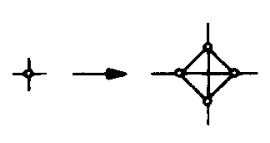

(b)

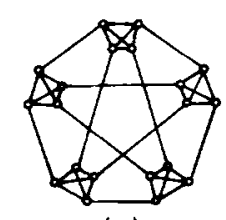

(c)
FIG. 1. (a) A complete graph on 5 points, zeroth order truncated 4-simplex lattice. (b) Replacement of single vertex by a complete 4 point graph. (c) Result of replacement on Fig. (a). First order truncated 4-simplex lattice. correctly from the scaling relations in terms of independently determinable critical exponents of the model. The quadratic interaction was chosen due to its simplicity。

In particular, the dimensionality of a lattice would not change if we introduced a second nearest neighbor spring interaction or any quadratic interaction of finite range for that matter. This may be proved rigorously using exact renormalization equations but is most easily seen by the application of first order perturbation theory. Let the first and second nearest neighbor spring constant be $J$ and $J^{\prime}$ respectively, $J^{\prime} \ll J$. The change in $\omega_{i}^{2}$, the frequency of the $i$ th mode is given by

$$
\frac{d \omega_{i}^{2}}{d J^{\prime}}=\alpha \omega_{i}^{2}+\text { higher order terms in } \omega_{i}^{2},
$$

where $\alpha$ is some constant depending on the lattice. This implies that the power law dependence of the density of states in the low frequency region remains unchanged.

\section{EXAMPLES OF LATTICE SYSTEMS WITH NONINTEGRAL DIMENSIONALITY}

In this section we describe the truncated $n$-simplex lattice and the modified rectangular lattice as examples of lattices with nonintegral dimensionality. Other lattices of this type are easy to construct. These lattices are so defined that exact renormalization equations may be written down for these systems.

The truncated $n$-simplex lattice is a simple generalization of the truncated tetrahedron lattice. The lattice is defined recursively. The graph of the zeroth order truncated $n$-simplex lattice is a complete graph on $(n+1)$ points. (A complete graph is a graph in which there exists a bond between every pair of lattice points.) The graph for the $(r+1)$ th order lattice is obtained by replacing each of the vertices of the $r$ th order graph by a complete graph on $n$ points. Each of the new $n$ points is connected to one of the lines coming to the original vertex. For the thermodynamic limit we let $r$ tend to infinity. In Fig. 1 we illustrate this for the case $n=4$.

The case $n=1$ corresponds to the uninteresting case of mutually disconnected pairs of points. $n=2$ corresponds to the linear chain. For $n=3$ we get the truncated tetrahedron lattice. For higher values of $n$ the lattices are nonplanar. It is easy to see that the $r$ th order truncated $n$ simplex lattice has $(n+1) n^{r}$ vertices and $\left[(n+1) n^{r+1}\right] / 2$ bonds. Each lattice point has a coordination number $n$. Though the lattice is multiply connected, it is still possible to disconnect an arbitrarily large set of points from the rest of the lattice by just deleting

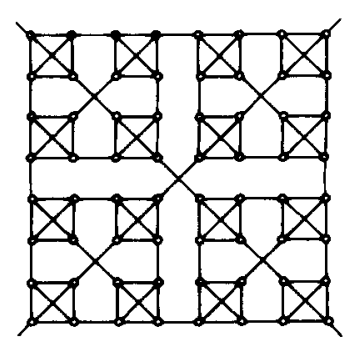

FIG. 2. A portion of the infinite truncated 4 -simplex lattice. 


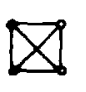

(a)

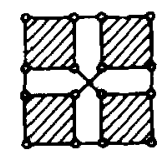

(b)

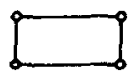

(a)

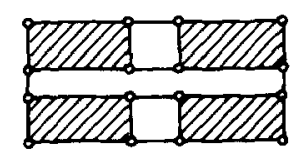

(b)
FIG. 3. (a) Graph of a first order square. (b) Schematic representation of the graph of the $(r+1)$ th order. The shaded squares denote the graph of $r$ th order squares of which only the corner vertices are shown.

$n$ bonds. We show later that the effective dimensionality of the truncated $n$-simplex lattice is $2 \log n / \log (n+2)$.

The truncated 4-simplex lattice may be more conveniently drawn as in Fig. 2 as a square lattice with bonds connecting at most the "next nearest neighbor " The quotation marks indicate that the word is used in the Euclidean sense. By our definition all pairs of points that have a bond in common are nearest neighbors. The construction of the graph is explained in Fig. 3. We define the first order square as a complete graph on four points. An $(r+1)$ th order square is obtained by joining four $r$ th order squares together by bonds such that each $r$ th order square is joined to the other $r$ th order squares by bonds connecting corner points and each $r$ th order square contributes one corner point to the $(r+1)$ th order square. The graph in Fig. 2 is a third order square. It is easy to convince oneself that the graph of the infinite order square is topologically equivalent to the infinite truncated 4-simplex graph. Thus we may generate the truncated 4 -simplex lattice from the graph of a two-dimensional infinite square lattice with "nearest neighbor" and "next nearest neighbor" bonds by selectively deleting some bonds. This deletion of bonds results in a change in the effective dimensionality of the lattice from 2 to $2 \log 4 / \log 6$.

We may similarly define a planar lattice to be called a modified rectangular lattice. This lattice has coordination number 3 and is multiply connected. The elementary cycles in the lattice are of length $4,8,16,32$, $\cdots$. The lattice is defined in Fig。 4. In this lattice the $r$ th order rectangle does not posses the full permutation symmetry between the corner lattice points as did the $r$ th order square in the previous case. This is because there is an asymmetry between the horizontal and vertical bonds and in the rth order rectangle $(r>1)$ there are more horizontal than vertical bonds. It is shown in Sec. III that the effective dimensionality of this lattice is $\frac{3}{2}$.

By varying $n$, in the truncated $n$-simplex lattice, we get lattices of effective dimensionality $0,1,2 \ln _{5} 3$, $2 \ln _{6} 4, \ldots$. However for any $n$ the dimensionality of the lattice is less than 2. The same is true for the modified rectangular lattice or some other lattices which may be defined recursively. However it is easy to obtain lattices of higher dimensionality. Given two lattices $L_{1}, L_{2}$ we define their direct product lattice $L=L_{1} \times L_{2}$ as follows: For each ordered pair $\left(l_{1}, l_{2}\right)$, where $l_{1}$ and $l_{2}$ are the lattice points in the lattices $L_{1}$ and $L_{2}$ respectively, we associate a unique lattice point $l \in L$. The point $l, l^{\prime} \in L$, where $l=\left(l_{1}, l_{2}\right), l^{\prime}=\left(l_{1}^{\prime}, l_{2}^{\prime}\right)$, are nearest neighbors of each others in $L$ iff
FIG. 4. (a) Graph of a first order rectangle. (b) Schematic representation of the graph of a $(r+1)$ th order rectangle. The shaded rectangles denote graphs of $r$ th order rectangles of which only the corner vertices are shown.

(i) $l_{1}=l_{1}^{\prime}$ and $\left(l_{2}\right.$ and $l_{2}^{\prime}$ are nearest neighbors in $\left.L_{2}\right)$ or

(ii) $l_{2}=l_{2}^{\prime}$ and $\left(l_{1}\right.$ and $l_{1}^{\prime}$ are nearest neighbors in $\left.L_{1}\right)$.

It is easy to see that effective dimensionality of $L$ is the sum of dimensionalities of $L_{1}$ and $L_{2}$. For example, the direct product of a linear chain and a square lattice is a simple cubic lattice of dimensionality $3=1+2$.

This gives us a whole class of lattices with nonintegral dimensionality so that the effect of changing dimensionality on critical exponents etc., may be studied with much more detail than has been possible so far. By forming the direct product of the modified rectangular lattice with itself we get a lattice of effective dimensions 3. Verification that critical exponents for, say, the Ising model on this lattice are the same as for the simple cubic lattice would be an important test of the usefulness and relevance of our definition of dimensionality and of the strength of the universality hypothesis.

\section{DETERMINATION OF THE EFFECTIVE DIMENSIONALITY}

In this section we determine the effective dimensionality of lattices defined in the previous section by determining their frequency spectrum. The procedure is equivalent to determining the low temperature specific heat behavior of a Debye solid on the lattice. If the low temperature specific heat is proportional to $T^{d}$ where $T$ is the temperature, we identify $d$ as the effective dimensionality of the lattice.

\section{A. The truncated $n$ simplex lattice}

We define the spherical model on this lattice by assigning a continuous spin $\chi_{i}\left(-\infty<\chi_{i}<\infty\right)$ to each of the sites $i$ of the $r$ th order truncated $n$-simplex lattice. The index $i$ goes from 1 to $N_{r}=(n+1) n^{r}$. The nearest neighbor sites are assumed to have a ferromagnetic Ising interaction of strength $J_{0}$ The spherical constraint is taken care of by introducing a Lagrange parameter $\lambda$ in the Hamiltonian so that the Hamiltonian of the system is

$$
H=(J / 2) \sum_{\text {nearest neighbors ij }}\left(\chi_{i}-\chi_{j}\right)^{2}+\left(\lambda_{J} / 2\right) \sum_{i=1}^{N_{r}} \chi_{i}^{2}
$$

We get the partition function as 


$$
Z_{r}(\lambda)=\left(\prod_{i=1}^{N_{r}} \int_{-\infty}^{+\infty} d \chi_{i}\right) \exp (-\beta H)
$$

where $\beta$ is the inverse temperature. This is a Gaussian integral and may be done easily, giving

$$
Z_{r}(\lambda)=\left(\frac{2 \pi}{\beta_{J}}\right)^{N_{r} 12} G_{r}(\lambda)
$$

with

$$
G_{r}(\lambda)=\prod_{i=1}^{N_{r}}\left(\lambda+\omega_{i}^{2}\right)^{-1 / 2}
$$

where $\omega_{i}^{2}$ are the normal mode frequencies of the model. The treatment of this model is quite parallel to that of the Ising model discussed in Ref. 1. In particular we may define the analog of star triangle and decoration transformations in the continuous spin case. The parameter $\lambda$ acts like some external field on each spin. These transformations follow from the identities

$$
\begin{aligned}
& \exp [-\left.\frac{1}{2} \sum_{i, j=1}^{n}\left(x_{i}-x_{j}\right)^{2}\right]=n \int_{-\infty}^{+\infty} \frac{d y}{\sqrt{2 \pi}} \\
& \times \exp \left[(-n / 2) \sum_{i=1}^{n}\left(y-x_{i}\right)^{2}\right] \\
& \int_{-\infty}^{+\infty} \frac{d \xi}{\sqrt{2 \pi}} \int_{-\infty}^{+\infty} \frac{d \eta}{\sqrt{2 \pi}} \exp \left(-\frac{n}{2}\left(x_{1}-\xi\right)^{2}-\frac{\lambda}{2} \xi^{2}-\frac{1}{2}(\xi-\eta)^{2}\right. \\
&\left.\quad-\frac{\lambda}{2} \eta^{2}-\frac{n}{2}\left(\eta-x_{2}\right)^{2}\right) \\
&=(\lambda+n)^{-1 / 2}(\lambda+n+2)^{-1 / 2} \\
& \quad \times \exp \left(-\frac{1}{2} \frac{n^{2}\left(x_{1}-x_{2}\right)^{2}}{(\lambda+n)\left(\lambda+\frac{1}{n+2}\right)}-\frac{1}{2} \frac{n \lambda\left(x_{1}^{2}+x_{2}^{2}\right)}{(\lambda+n)}\right)
\end{aligned}
$$

These identities are applied as follows. We group the $N_{r}$ spins into $N_{r} / n$ groups of $n$ spins each, such that each spin interacts with every other spin of the same group with interaction strength 1 in scaled units. Then we introduce a new spin $y_{j}$ for each group $j$ and couple it to each of the spins of its group with interaction strength $n$. The original $n(n-1) / 2$ bonds within each group are deleted and replaced by $n$ new bonds all connecting the new spins $y_{j}$ to other spins (Fig. 5). Identity (5) guarantees that partition function remains unchanged.

The second identity corresponds to the dedecoration transformation of Fisher [Fig. 6]. We integrate over all the original spins $\chi_{i}$. This leaves us with the new spins $y_{j}$ which form an $(r-1)$ th order truncated $n$-sim-

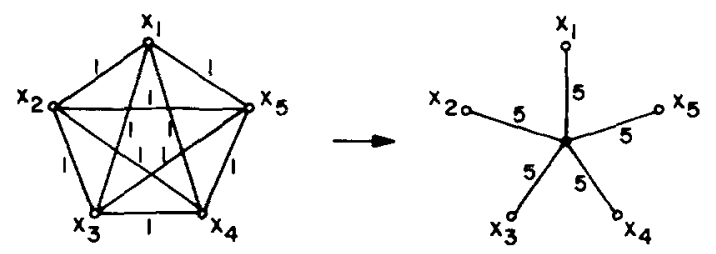

FIG. 5. An example of the generalized star triangle transformation with $n=5$. plex lattice and all the spins $y_{j}$ interact with their nearest neighbor spins on this lattice by quadratic interaction. By a scale transformation on the spin $y_{j}$ this new interaction may be converted into an interaction of unit strength.

Applying these transformations we find that

$G_{r}(\lambda)=[(\lambda+n)(\lambda+n+2)]^{-N_{r} / 4+N_{r} / 2 n} G_{r-1}\left(\lambda^{2}+n \lambda+2 \lambda\right)$.

Define

$$
F(\lambda)=\lim _{r \rightarrow \infty} \ln G_{r}(\lambda) / N_{r}
$$

We get

$$
F(\lambda)=\frac{n-2}{4 n} \ln \frac{1}{(\lambda+n)(\lambda+n+2)}+\frac{1}{n} F\left(\lambda^{2}+n \lambda+2 \lambda\right) 。
$$

The frequency spectrum of the model may be determined from this functional equation for the characteristic function $F(\lambda)$. Details of the calculation are given in the Appendix. We note that $\lambda=0$ is a fixed point of the transformation; from $\mathrm{Eq}_{0}$ (8), putting $\lambda=0$, we get

$$
F(0)=\frac{n-2}{4(n-1)} \ln \frac{1}{n(n+2)} .
$$

From Eq. (A1) of the Appendix, it is easily seen that for small $\lambda>0, F(\lambda)$ has an asymptotic expansion of the form $F(\lambda)=F(0)+A \lambda^{d / 2}+$ (higher order in $\lambda$ ), where $d$ is the dimensionality of the lattice. Substituting in Eq. (8) we get

$$
d=2 \log _{n+2} n \text {. }
$$

It is interesting to note that this result may be obtained without the complicated analysis of Eq. (8) given in the Appendix.

\section{B. The modified rectangular lattice}

For this lattice we wish to determine a functional equation of the same general form as Eq. (8) from which the dimensionality of the lattice may be deduced. The functional equation is however much more complicated and involves five variables instead of one.

We have

$$
H=(J / 2) \sum_{(i j)}\left(\chi_{i}-\chi_{j}\right)^{2}+(\lambda J / 2) \sum_{i=1}^{N_{r}} \chi_{i}^{2},
$$

where the sum over $(i j)$, extends over all the nearest neighbors. We consider a more general form of the Hamiltonian and allow different interaction strengths and more than nearest neighbor interaction between the spins belonging to the same first order rectangle. We write

$$
H=\frac{1}{2} \sum_{(i j)} J_{i j}\left(\chi_{i}-\chi_{j}\right)^{2}+(\lambda J / 2) \sum_{i} \chi_{i}^{2},
$$

where $J_{i j}=0$ unless $i$ and $j$ are nearest neighbor or they belong to the same first order rectangle. $J_{i j}$ may take

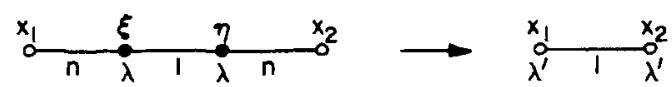

FIG. 6. The dedecoration transformation. Spins $\xi$ and $\eta$ are integrated over. 


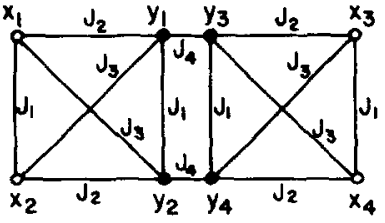

FIG. 7. Coupling constants for the modified rectangular lattice. The spins $y_{1}, y_{2}, y_{3}$, $y_{4}$ are integrated over. four different values $J_{1}, J_{2}, J_{3}$, or $J_{4}$. These interactions are depicted in Fig. 7. $J_{1}, J_{2}$, and $J_{3}$ are respectively the vertical, horizontal, and diagonal bond strengths if $i$ and $j$ belong to the same first order rectangle. $J_{4}$ is the interaction strength if $i j$ are nearest neighbors but do not belong to the same first order rectangle.
The renormalization transformation consists of integrating over the spins $y_{1}, y_{2}, y_{3}$, and $y_{4}$ and similar spins in other rectangles. This reduces the number of degrees of freedom in the Hamiltonian by a factor of 2 . The integration over $y_{i}(i=1,4)$ gives us an effective interaction between $x_{i}(i=1$ to 4$)$ and since the interaction is quadratic, it may again be expressed in the form (11) except for new values of the coupling constants $J_{1}, J_{2}, J_{3}$. The value of $J_{4}$ remains the same. The spins $x_{i}(i=1$ to 4$)$ may be said to form a higher order spin block (second order). We note that an $r$ th order rectangle is, by definition, a $(2 r-1)$ th order spin block. The most general form of the weight of the $r$ th order spin block with corner spins $x_{1}, x_{2}, y_{1}, y_{2}$ and all other spins integrated over is

$$
W^{(r)}\left(x_{1}, x_{2} ; y_{1}, y_{2}\right)=C^{(r)} \exp \left[\begin{array}{l}
-\frac{\beta J_{1}^{(r)}}{2}\left\{\left(x_{1}-x_{2}\right)^{2}+\left(y_{1}-y_{2}\right)^{2}\right\}-\frac{\beta J_{2}^{(r)}}{2}\left\{\left(x_{1}-y_{1}\right)^{2}+\left(x_{2}-y_{2}\right)^{2}\right\} \\
-\frac{\beta J_{3}^{(r)}}{2}\left\{\left(x_{1}-y_{2}\right)^{2}+\left(x_{2}-y_{1}\right)^{2}\right\}-\frac{\beta J \lambda^{(r)}}{2}\left\{x_{1}^{2}+x_{2}^{2}+y_{1}^{2}+y_{2}^{2}\right\}
\end{array}\right] .
$$

We also have the recursion relation (Fig. 7)

$$
\begin{aligned}
& W^{(r+1)}\left(x_{1}, x_{3} ; x_{2}, x_{4}\right) \\
& =\iiint \int_{-\infty}^{+\infty} d y_{1} d y_{2} d y_{3} d y_{4} W^{(r)}\left(x_{1}, x_{2} ; y_{1}, y_{2}\right) \\
& \quad \times W^{(r)}\left(y_{3}, y_{4} ; x_{3}, x_{4}\right) \exp \left(-\frac{\beta J_{4}}{2}\left\{\left(y_{1}-y_{3}\right)^{2}+\left(y_{2}-y_{4}\right)^{2}\right\}\right) .
\end{aligned}
$$

The integrations in this equation are Gaussian and may be carried out easily. This gives us the equations for the new coupling constants

$$
\begin{aligned}
& C^{(r+1)}=\left(C^{(r)}\right)^{2}(2 \pi)^{2} \beta^{2}\left(\lambda^{(r)} J+J_{2}^{(r)}+J_{3}^{(r)}\right)^{-1 / 2} \\
& \times\left(\lambda^{(r)} J+2 J_{1}^{(r)}+J_{2}^{(r)}+J_{3}^{(r)}\right)^{-1 / 2} \\
& \times\left(\lambda^{(r)} J+J_{2}^{(r)}+J_{3}^{(r)}+2 J_{4}\right)^{-1 / 2} \\
& \times\left(\lambda^{(r)} J+2 J_{1}^{(r)}+2 J_{4}+J_{2}^{(r)}+J_{3}^{(r)}\right)^{-1 / 2} \text {, } \\
& \lambda^{(r+1)}=\lambda^{(r)}\left(\lambda^{(r)} J+2 J_{2}^{(r)}+2 J_{3}^{(r)}\right)\left(\lambda^{(r)} J+J_{2}^{(r)}+J_{3}^{(r)}\right)^{-1} \text {, } \\
& \lambda^{(r+1)} J+2 J_{1}^{(r+1)}+2 J_{3}^{(r+1)} \\
& =\lambda^{(r)} J+J_{2}^{(r)}+J_{3}^{(r)}-\left(J_{2}^{(r)}+J_{3}^{(r)}\right)^{2}\left(\lambda^{(r)} J+2 J_{4}\right. \\
& \left.+J_{2}^{(r)}+J_{3}^{(r)}\right)^{-1} \\
& \lambda^{(r+1)} J+2 J_{2}^{(r+1)}+2 J_{3}^{(r+1)} \\
& =\left(\lambda^{(r)} J+2 J_{1}^{(r)}+2 J_{3}^{(r)}\right)\left(\lambda^{(r)} J+2 J_{1}^{(r)}+2 J_{2}^{(r)}\right) \\
& \times\left(\lambda^{(r)} J+2 J_{1}^{(r)}+J_{2}^{(r)}+J_{3}^{(r)}\right)^{-1}, \\
& =\left[( \lambda ^ { ( r ) } J + 2 J _ { 1 } ^ { ( r ) } + 2 J _ { 3 } ^ { ( r ) } ) \left(\lambda^{(r)} J+2 J_{1}^{(r)}+2 J_{2}^{(r)}+2 J_{4}\right.\right. \\
& \left.\times\left(\lambda^{(r)} J+2 J_{1}^{(r)}+J_{2}^{(r)}+J_{3}^{(r)}\right)\right] \\
& {\left[\lambda^{(r)} J+2 J_{4}+2 J_{1}^{(r)}+J_{2}^{(r)}+J_{3}^{(r)}\right]^{-1} .}
\end{aligned}
$$

If the free energy per spin of the Hamiltonian given by $\mathrm{Eq}$. (11) is given by $F\left(J_{1}, J_{2}, J_{3}, J_{4}, \lambda\right)$ we see that the renormalization transformation gives us the equation

$F\left(J_{1}, J_{2}, J_{3}, J_{4}, \lambda\right)=\frac{1}{8} \ln \frac{C^{(r+1)}}{\left(C^{(r)}\right)^{2}}+\frac{1}{2} F\left(J_{1}^{\prime}, J_{2}^{\prime}, J_{3}^{\prime}, J_{4}^{\prime}, \lambda^{\prime}\right)$,

where $J_{1}^{\prime}, J_{2}^{\prime}, J_{3}^{\prime}, J_{4}, \lambda^{\prime}$ are given by Eqs. $(14 \mathrm{~b})-(14 \mathrm{e})$. To get the free energy per spin of the original modified rectangular model we put $J_{1}=J_{2}=J_{4}=J, J_{3}=0$. We note that $\lambda=0$ is a fixed point of the renormalization transformation. The free energy per spin is a singular function of $\lambda$ near $\lambda=0$ and has an asymptotic expansion of the form $(\lambda>0)$.

$$
\begin{aligned}
F\left(J_{1}, J_{2}, J_{3}, J_{4}, \lambda\right)= & F\left(J_{1}, J_{2}, J_{3}, J_{4}, 0\right) \\
& +\lambda^{d / 2} G\left(J_{1}, J_{2}, J_{3}, J_{4}\right) \\
& + \text { higher order terms in } \lambda,
\end{aligned}
$$

We wish to determine the behavior of $F$ for small $\lambda$. However, the value of $\lambda$ increases with iteration approximately as $\lambda^{(r+1)} \approx 2 \lambda^{(r)}$ as is evident from Eq. (14b). Also values of $J_{1}, J_{2}, J_{3}$ decrease with iteration. We choose initial $\lambda$ sufficiently small and $r$ sufficiently large so that

$$
\lambda^{(r)} J \ll J_{1}^{(r)}, J_{2}^{(r)}, J_{3}^{(r)} \ll J_{4} \text { 。 }
$$

Then the recursion equations (14) may be approximated as

$$
\begin{aligned}
& \lambda^{(r+1)} \approx 2 \lambda^{(r)} \\
& J_{1}^{(r+1)}+J_{3}^{(r+1)} \approx \frac{1}{2}\left(J_{2}^{(r)}+J_{3}^{(r)}\right) \\
& J_{2}^{(r+1)}+J_{3}^{(r+1)} \approx 2\left(J_{1}^{(r)}+J_{2}^{(r)}\right)\left(J_{1}^{(r)}+J_{3}^{(r)}\right)\left(2 J_{1}^{(r)}+J_{2}^{(r)}+J_{3}^{(r)}\right)^{-1}
\end{aligned}
$$

$J_{1}^{(r+1)}+J_{2}^{(r+1)} \approx J_{1}^{(r)}+\frac{1}{2} J_{2}^{(r)}+\frac{1}{2} J_{3}^{(r)}$

Now if we write $F^{\text {singular }}\left(J_{1}, J_{2}, J_{3}, J_{4}, \lambda\right)$ as the singular part of free energy near $\lambda=0$, we get from Eq. (15) 


$$
\begin{aligned}
& F^{\otimes 1 \text { agular }}\left(J_{1}^{(r)}, J_{2}^{(r)}, J_{3}^{(r)}, J_{4}, \lambda^{(r)}\right) \\
& \quad \approx \frac{1}{2} F^{\text {singular }}\left(J_{1}^{(r+1)}, J_{2}^{(r+1)}, J_{3}^{(r+1)}, J_{4}, \lambda^{(r+1)}\right) .
\end{aligned}
$$

It can be shown that Eqs. (18b)-(18d) imply that $J_{2}^{(r) /}$ $J_{1}^{(r)}$ and $J_{3}^{(r)} / J_{1}^{(r)}$ tend to the limiting ratios $\left(2^{1 / 3}+2^{-1 / 3}\right)$ and $\left(2^{-1 / 3}\right)$ geometrically with $r$. Hence for large enough $r$ the Eqs. (18a)-(18d) have the form

$$
\begin{aligned}
& \lambda^{(r+1)} \approx 2 \lambda^{(r)}, \\
& J_{1}^{(r+1)} \approx 2^{-1 / 3} J_{1}^{(r)}, \\
& J_{2}^{(r+1)} \approx 2^{-1 / 3} J_{2}^{(r)}, \\
& J_{3}^{(r+1)} \approx 2^{-1 / 3} J_{3}^{(r)} .
\end{aligned}
$$

Now since the variable $J_{2}^{(r)}$ and $J_{3}^{(r)}$ are asympotically proportional to $J_{1}^{(r)}$, the se variables are unnecessary for our discussion. Any one of the variables $J_{1}^{(r)}, J_{2}^{(r)}$, $J_{3}^{(r)}$ is adequate for the discussion of critical properties of the model. These variables provide the relevant scale for our problem. We assume that $F^{\text {singular }}\left(J_{1}, J_{2}, J_{3}, J_{4}, \lambda\right)$ $\approx A\left(\lambda J_{4} / J_{1}\right)^{d / 2}$ where $A$ is some constant. Substituting this in Eq. (19) and with the help of Eqs. (20a) and (20b) we find that $d=\frac{3}{2}$ which is the promised result.

\section{ANALYSIS OF THE CRITICAL BEHAVIOR OF SOME OTHER HAMILTONIANS}

In the previous section the nearest neighbor harmonic interaction model (or equivalently the spherical model) on the truncated $n$-simplex lattice and the modified rectangular lattice was analyzed. Nelson and Fisher ${ }^{1}$ have analyzed the behavior of the Ising model on the truncated tetrahedron lattice and determined the behavior of specific heat and susceptibility as a function of temperature. In the following we sketch the behavior of some other Hamiltonians on these lattices. Due to the special structure of these lattices, exact renormalization equations may be written down involving only a finite number of coupling constants. For definiteness we shall consider only the truncated 3-simplex lattice. Other lattices may be treated similarly and so long as $d<2$ the qualitative behavior of the lattices is quite similar and is characterized by the absence of phase transitions at any finite temperature. Lattices with $d>2$ are more interesting because they show phase transitions but the exact renormalization equations are much more complicated and are difficult to analyze. We confine ourselves to the nearest neighbor interactions for simplicity。

\section{A. The classical $X Y$ model}

In the classical XY model, a spin direction $\theta_{i}$, $0<\theta_{i} \leqslant 2 \pi$, is assigned to each site $i$ of the lattice. The Hamiltonian of the system is given by

$$
H=-J \sum_{(i j)} \cos \left(\theta_{i}-\theta_{j}\right)-h \sum_{i} \cos \theta_{i},
$$

where the summation over $i j$ extends over all pairs of nearest neighbor sites. The corresponding partition function is given by

$$
Z=\prod_{i}\left(\int_{0}^{2 r} \frac{d \theta_{i}}{2 \pi}\right) \exp [-\beta H] .
$$

First consider the case of zero magnetic field. We

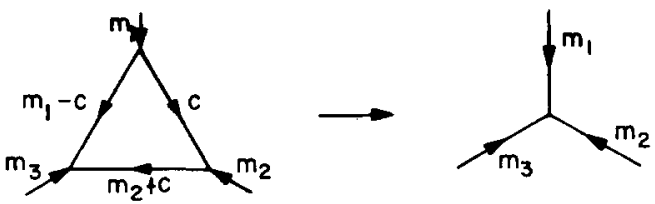

FIG. 8. The renormalization transformation for the XY model on the truncated 3-simplex lattice. The three vertices in the left figure are replaced by a single vertex.

write

$$
\exp \left[\beta J \cos \left(\theta_{i}-\theta_{j}\right)\right]=\sum_{n_{i j}=-\infty}^{+\infty} I_{n_{i j}}(\beta J) \exp \left[i n_{i j}\left(\theta_{i}-\theta_{j}\right)\right],
$$

where $I_{m}(x)$ is the modified Bessel function of $x$ of order $m$ and express $Z$ as a summation over all possible values of $\left(n_{i j}\right)$. With each term of this summation we associate an arrow configuration on the bonds of the lattice. The arrow configuration has $n_{i j}$ arrows going from site $i$ to site $j$ for all nearest neighbor sites $i$ and $j$ with the convention $n_{i j}=-n_{j i}$, Thus the partition function is expressed as a sum over all the possible arrow configurations. The weight of a bond.with $r$ arrows is $I_{r}(\beta J)$, Integration over $\theta_{i}$ are trivial and show that only those configurations of arrows contribute to the partition sum in which the total number of arrows going into any site is zero.

Equivalently we may assign a weight $T\left(m_{1}, m_{2}, m_{3}\right)$ to a vertex with $m_{1}, m_{2}, m_{3}$ arrows going into it from its three nearest neighbors. If $m_{1}+m_{2}+m_{3} \neq 0$ we set the corresponding vertex weight $T\left(m_{1}, m_{2}, m_{3}\right)=0$ 。

The renormalization procedure for the truncated 3 simplex lattice consists of replacing a group of three vertices by a single vertex and is illustrated in Fig, 8 . If $T^{(r)}\left(m_{1}, m_{2}, m_{3}\right)$ denotes the vertex weight after $r$ iterations of the renormalization transformation, the renormalization equations are given by

$$
\begin{aligned}
T^{(r+1)} & \left(m_{1}, m_{2}, m_{3}\right) \\
= & \sum_{c=-\infty}^{+\infty} T^{(r)}\left(m_{1}, c-m_{1},-c\right) T^{(r)}\left(m_{2}, m_{1}-c, c+m_{3}\right) \\
& \times T^{(r)}\left(m_{3}, c,-c-m_{3}\right) .
\end{aligned}
$$

The starting values of vertex weights are

$$
T^{(0)}\left(m_{1}, m_{2},-m_{1}-m_{2}\right)=\left\lfloor I_{m_{1}}(\beta J) I_{m_{2}}(\beta J) I_{-m_{1}-m_{2}}(\beta J)\right]^{1 / 2}
$$

The free energy per site is given by

$$
f(\beta)=-\frac{1}{\beta} \lim _{r \rightarrow \infty} \frac{1}{3^{r}} \ln T^{(r)}(0,0,0) .
$$

It is easy to verify that Eqs. (24) have only one attractive fixed point given by

$$
T^{(r)}\left(m_{1}, m_{2}, m_{3}\right) / T^{(r)}(0,0,0)=\delta_{m_{1}, 0^{\delta}} m_{2,0} \delta_{m_{3}, 0} .
$$

This corresponds to spin directions at large distances being uncorrelated and hence to the absence of spontaneous magnetization. If the temperature is very low, $\beta J \gg\left(m_{1}^{2}+m_{2}^{2}+m_{3}^{3}\right)$, we have 


$$
\begin{aligned}
& T^{(0)}\left(m_{1}, m_{2},-m_{1}-m_{2}\right) \\
& \quad \approx A^{(0)} \exp \left[-\left\{m_{1}^{2}+m_{2}^{2}+\left(-m_{1}-m_{2}\right)^{2}\right\} / 4 \beta J\right] .
\end{aligned}
$$

Then from Eq. (24) we get

$$
\begin{aligned}
& T^{(1)}\left(m_{1}, m_{2},-m_{1}-m_{2}\right) \\
& \quad \approx A^{(1)} \exp \left[-5\left\{m_{1}^{2}+m_{2}^{2}+\left(-m_{1}-m_{2}\right)^{2}\right\} / 12 \beta J\right],
\end{aligned}
$$

where $A^{(0)}$ and $A^{(1)}$ are some constants. This shows that the renormalization equation for the temperature is

$$
\beta^{\prime}=\frac{3}{5} \beta_{\text {。 }}
$$

Now let us introduce a small external field $h, \beta h \ll 1$. It is easy to see that the vertex weights $T\left(m_{1}, m_{2}, m_{3}\right)$ are no longer zero if $m_{1}+m_{2}+m_{3} \neq 0$. To first order in $\beta h$, and for $\beta J \gg\left(m_{1}^{2}+m_{2}^{2}+m_{3}^{2}\right)$ the other nonzero vertex weights are

$$
\begin{aligned}
& T^{(0)}\left(m_{1}, m_{2}, m_{3}\right)=\beta h A^{(0)} \exp \left[-\left\{m_{1}^{2}+m_{2}^{2}+m_{3}^{2}\right\} / 4 \beta J\right] \\
& \text { for } m_{1}+m_{2}+m_{3}= \pm 1 .
\end{aligned}
$$

After one renormalization transformation, these may be shown to transform to

$T^{(1)}\left(m_{1}, m_{2}, m_{3}\right)=3 \beta h A^{(1)} \exp \left[-5\left\{m_{1}^{2}+m_{2}^{2}+m_{3}^{2}\right\} / 12 \beta J\right]$

for $m_{1}+m_{2}+m_{3}= \pm 1$.

Thus we see that to the lowest order in $h$, the external field is transformed according to the renormalization equation

$$
\beta^{\prime} h^{\prime}=3 \beta h \text {. }
$$

Using Eqs. (30) and (33), it is easy to determine the behavior of susceptibility as a function of temperature. Let $g(\beta, h)$ be the singular part of the logarithm of partition function per spin. By the renormalization transformation

$$
g(\beta, h)=\frac{1}{3} g\left(\frac{3}{5} \beta, 5 h\right),
$$

and by the susceptibility

$$
\begin{aligned}
\chi(\beta) & =-\left.\frac{1}{\beta} \frac{\partial^{2} g(\beta, h)}{\partial h^{2}}\right|_{h=0}=-\left.\frac{5}{\beta^{\prime}} \frac{\partial^{2} g\left(\beta^{\prime}, h^{\prime}\right)}{\partial h^{\prime 2}}\right|_{h^{\prime}=0} \\
& =5 \chi\left(\frac{3}{5} \beta\right) .
\end{aligned}
$$

Hence if $\chi(\beta) \sim \beta^{\bar{\gamma}}$ we get

$$
\bar{\gamma}=\ln 5 /(\ln 5-\ln 3) \text {. }
$$

Similar analysis of the truncated $n$-simplex lattice for arbitrary $n$ shows that in general, for $d<2$

$$
\bar{\gamma}=2 /(2-d) \text {. }
$$

The specific heat tends to simple harmonic oscillator value $\frac{1}{2} K_{B}$ as the temperature tends to zero.

The exponent $\bar{\gamma}$ in Eq. (37) should not be identified with the critical exponent $\gamma$ which appears in the scaling relations and specifies the divergence of susceptibility as a function of temperature slightly away from the critical point. The reason is the arbitrariness in the definition of critical exponents when the critical temperature is zero. The procedure of determining critical exponents in terms of the divergence of correlation length ${ }^{1}$ is of doubtful validity here because the lattice is not translationally invariant and the "correlation length" is a function of position. Different definitions of the "averaged correlation length" may well give rise to different dependence on temperature. In general, it is difficult to find a useful definition of the correlation length for the spatially inhomogeneous and highly anisotropic lattices studied here. Consequently the critical exponents $\nu$ and $\eta$ are undefined even if the transition temperature is finite. (To define them in terms of scaling relations would be begging the question.) The critical exponents like $\alpha, \beta, \gamma, \delta$, etc., which may be defined by differentiation of thermodynamic quantities are however unambiguously defined for nonzero transition temperatures.

\section{B. The Fortuin-Kasteleyn cluster model}

The cluster model was introduced by Fortuin and Kasteleyn ${ }^{5-7}$ It is defined in terms of a parameter $k$ and includes as special cases the percolation model $(\kappa=1)$, the Ising model $(\kappa=2)$, resistive networks $(\kappa=0)$, and the $n$-state Potts model $(\kappa=n)$, etc。

The partition function of the cluster model for any given graph is given by

$$
Z(p, \kappa)=\sum_{C} p^{r(c)}(1-p)^{N-r(C)} \kappa^{\gamma(C)},
$$

where $N$ is the total number of bonds in the graph. The summation extends over all the possible configurations $C$ of "occupied" or "unoccupied" states of bonds on the lattice, $r(C)$ is the number of occupied bonds in the configuration $C$ and $\gamma(C)$ is the number of distinct clusters in the configuration $C$, Fortuin and Kastelyn have called this the random cluster model. However the model is completely specified by a Hamiltonian which has a term proportional to the number of occupied bonds and a term proportional to the total number of clusters, It is thus no more random than any other statistical mechanical system and the adjective "random" is unnecessary

Due to its very general nature, the cluster model is of great interest in statistical physics. However, for arbitrary values of $\kappa$ the model has not been solved even in two dimensions. We sketch below the renormalization group treatment of this model for the truncated tetrahedron lattice. It is shown that the model does not show any phase transition for any values of $k$ and $p$ such that $\kappa \cdot 0,0 \leqslant p \leqslant 1$.

We define the restricted partition functions $Z^{(r)}(\alpha|\beta| \gamma), Z^{(r)}(\alpha \beta \mid \gamma), Z^{(r)}(\beta \gamma \mid \alpha), Z^{(r)}(\alpha \gamma \mid \beta)$, and $Z^{(r)}(\alpha \beta \gamma)$. Here $Z^{(r)}(\alpha|\beta| \gamma)$ is the partition function of the $r$ th order triangle whose corner vertices are $\alpha, \beta$, and $\gamma$ and the summation corresponds to all configurations of edges within the triangle subject to the constraint that no sequence of occupied bonds within the $r$ th order triangle connects any two of the vertices $\alpha, \beta, \gamma_{0} Z^{(r)}(\alpha \beta \gamma)$ is defined similarly except that in this case $\alpha, \beta$, and $\gamma$ are connected together by bonds lying within the $r$ th order triangle. By symmetry between the corner vertices $\alpha, \beta$, and $\gamma$ we have

$$
Z^{(r)}(\alpha \beta \mid \gamma)=Z^{(r)}(\beta \gamma \mid \alpha)=Z^{(r)}(\alpha \gamma \mid \beta)=Z_{2}^{(r)} \text { (say) }
$$

and we write 


$$
\begin{gathered}
Z^{(r)}(\alpha|\beta| \gamma)=Z_{1}^{(r)}, \\
Z^{(r)}(\alpha \beta \gamma)=Z_{3}^{(r)} .
\end{gathered}
$$

It is quite straightforward though tedious to write down the expressions for $Z_{1}^{(r+1)}, Z_{2}^{(r+1)}, Z_{3}^{(r+1)}$ in terms of $Z_{1}^{(r)}, Z_{2}^{(r)}, Z_{3}^{(r)}$ by summing over the $2^{3}$ possible states of the three bonds that connect the $r$ th order triangles to form the $(r+1)$ th order triangle and grouping the terms that correspond to the same connectivity structure of the $(r+1)$ th order triangle together. The result is

$$
\begin{aligned}
Z_{1}^{(r+1)}= & q^{3}\left(Z_{1}+3 Z_{2}+Z_{3}\right)^{3}+3 p q^{2} \kappa^{-1}\left(Z_{1}+3 Z_{2}+Z_{3}\right) \\
& \times\left(Z_{1}+2 Z_{2}\right)\left(Z_{1}+4 Z_{2}+2 Z_{3}\right)+3 p^{2} q \kappa^{-2} \\
& \times\left\{\left(Z_{1}+2 Z_{2}\right)^{3}+3\left(Z_{1}+2 Z_{2}\right)^{2}\left(Z_{2}+Z_{3}\right)+Z_{1}\left(Z_{2}+Z_{3}\right)^{2}\right\} \\
& +p^{3} \kappa^{-3}\left\{\kappa\left(3 Z_{3} Z_{2}^{2}+Z_{2}^{3}\right)+14 Z_{2}^{3}+12 Z_{1} Z_{2} Z_{3}\right. \\
& \left.+24 Z_{2}^{2} Z_{1}+3 Z_{1}^{2} Z_{3}+9 Z_{1}^{2} Z_{2}+Z_{1}^{3}\right\} \\
Z_{2}^{(r+1)}= & q^{2} p \kappa^{-1}\left(Z_{1}+3 Z_{2}+Z_{3}\right)\left(Z_{2}+Z_{3}\right)^{2}+p^{2} q \kappa^{-2}\left(Z_{2}+Z_{3}\right)^{2} \\
& \times\left(2 Z_{1}+7 Z_{2}\right)+p^{3} \kappa^{-3}\left\{\kappa Z_{3}^{2} Z_{2}+7 Z_{2}^{2} Z_{3}+Z_{3}^{2} Z_{1}\right. \\
& \left.+2 Z_{1} Z_{2} Z_{3}+4 Z_{2}^{3}+Z_{1}^{2} Z_{3}\right\} \\
Z_{3}^{(r+1)}= & 3 p^{2} q \kappa^{-2}\left(Z_{2}+Z_{3}\right)^{2} Z_{3}+p^{3} \kappa^{-3}\left(\kappa Z_{3}^{3}+6 Z_{2} Z_{3}^{2}+3 Z_{2}^{2} Z_{3}\right),
\end{aligned}
$$

where we have put $q=1-p$ and $Z_{i}^{(r)}=Z_{i}, i=1,2,3$. If $q>0$, analysis of these equations shows that there exists only one fixed point which is attractive and corresponds to $Z_{2} / Z_{1}=Z_{3} / Z_{1}=0$. This implies absence of phase transitions at any finite temperature. For small $q$ and for small $r, Z_{3}^{(r)}$ is much larger than $Z_{2}^{(r)}$ or $Z_{1}(r)$. If we renormalize the $Z_{i}$ after each iteration so that $Z_{1}^{(r)}+3 Z_{2}^{(r)}+Z_{3}^{(r)}=1$ we get to the lowest order in $q$

$$
\begin{aligned}
& Z_{1}^{(r)}=\kappa^{2} q^{3}, \\
& Z_{2}^{(r)}=\kappa q^{2} r, \\
& Z_{3}^{(r)}=1-3 q^{2} \kappa r .
\end{aligned}
$$

From this we may deduce that for small $q$ the mean size of one cluster (which corresponds to the susceptibility for the Ising model) is approximately given by $3^{a / x a^{2}}$ where $a$ is some absolute constant. For the Ising model, this result has already been obtained by Nelson and Fisher. We need only note the correspondence $q=e^{-\beta J} /\left(e^{\beta J}+e^{-\beta J}\right)$ and $k=2$ to specialize our result to the Ising model of interaction strength $J_{\text {。 }}$

To summarize, in this paper we have introduced a class of lattices of effectively nonintegral dimensionality. The lattices are not translationally invariant but sufficiently regular so that the existence of thermodynamic limit is assured for most physically interesting Hamiltonians. The procedure of determining critical exponents as a function of dimensionality was illustrated in Sec. IV where the classical $X Y$ model was analyzed for $d<2$. For lattices with $d>2$, exact analysis of thermodynamic properties is difficult; but approximate renormalization group equations or numerical extrapolation methods like series expansions may be used to determine the value of critical exponents. This seems to be a promising field for further investigation.

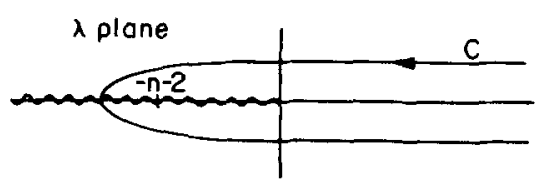

FIG. 9. The complex $\lambda$ plane showing the curving $C$ and the branch cut along the negative real axis.

\section{APPENDIX}

We have the equation

$$
F(\lambda)=\frac{n-2}{4 n} \ln \{(\lambda+n)(\lambda+n+2)\}^{-1}+\frac{1}{n} F\left(n \lambda+2 \lambda+\lambda^{2}\right)
$$

This equation is valid for all real $\lambda>0$. As $\lambda$ tends to infinity $F(\lambda)$ tends to $-\frac{1}{2} \ln \lambda$. This condition specifies $F(\lambda)$ completely for all real positive $\lambda$ when combined with Eq. (8). From Eq。(4) we may write

$$
F(\lambda)=-\frac{1}{2} \int_{0}^{\omega_{\max }^{2}} d \omega^{2} D\left(\omega^{2}\right) \ln \left(\omega^{2}+\lambda\right),
$$

where $D\left(\omega^{2}\right) d \omega^{2}$ is the fractional number of modes in the frequency range $\omega^{2}$ and $\omega^{2}+d \omega^{2}$. From Eq. (A1) we may define $F(\lambda)$ as an analytic function in $\lambda$ over the entire complex $\lambda$ plane. The function has logarithmic branch points on the negative real axis and consists of many sheets. Consider the sheet in which $F(\lambda)$ is real for all real positive $\lambda$. We make the branch cut along the negative real line. Then it is easy to see that

$$
\begin{aligned}
& H\left(\omega^{2}\right) \stackrel{\text { der }}{=}-\frac{2}{\pi} \operatorname{Im} F\left(-\omega^{2}+i_{\epsilon}\right), \text { for all } \omega^{2}, \\
& =\int_{0}^{\omega^{2}} d \omega^{\prime 2} D\left(\omega^{\prime 2}\right), \quad \text { for } \omega^{2}>0 .
\end{aligned}
$$

Consider $\lambda=\lambda_{R}+i \epsilon$ where $\lambda_{R}$ is real. As we vary $\lambda_{R}$ from $+\infty$ to $-\infty$, the function $\left(\lambda^{2}+n \lambda+2 \lambda\right)$ traces out the curve $C$ shown in Fig. 9. For $\lambda_{R}<-(n+2) / 2$ the curve $C$ crosses over the negative real axis and goes into a different sheet. The value of the function $F(\lambda)$ on this sheet differs from its value on the original sheet by an additive imaginary constant. Taking the imaginary part of the Eq. (8) it is easy to show that

$$
\begin{aligned}
0<\omega^{2}<\frac{n+2}{2}, \quad H\left(\omega^{2}\right) & =\frac{1}{n} H\left(n \omega^{2}+2 \omega^{2}-\omega^{4}\right) \\
\frac{n+2}{2}<\omega^{2}<\infty, \quad H\left(\omega^{2}\right)= & \frac{n-2}{2 n}\left\{E\left(\omega^{2}-n\right)+E\left(\omega^{2}-n-2\right)\right\} \\
& +\frac{2}{n}-\frac{1}{n} H\left(n \omega^{2}+2 \omega^{2}-\omega^{4}\right)
\end{aligned}
$$

where $E(x)$ is the unit step function which is zero for negative arguments and +1 for positive arguments. From Eq. (A3) it is easy to see that $H\left(\omega^{2}\right)$ is a nonnegative monotonically increasing function of $\omega^{2}$, and we have

$$
\begin{aligned}
& H\left(\omega^{2}\right)=0, \text { for }-\infty<\omega^{2} \leqslant 0, \\
& H\left(\omega^{2}\right)=1, \text { for } \infty>\omega^{2}>n+2 .
\end{aligned}
$$

We shall determine the function $H\left(\omega^{2}\right)$ in the unknown range $0<\omega^{2}<(n+2)$ by repeated application of Eqs. (A4) and (A5). 
Consider $\omega_{1}^{2}<\omega^{2}<n$ where $(n+2) \omega_{1}^{2}-\omega_{1}^{4}=(n+2)$. Then from Eq. (A7) we get $H\left(\omega^{2}(n+2)-\omega^{4}\right)=1$ and hence by using Eqs. (A4) and (A5) we get

$$
H\left(\omega^{2}\right)=\frac{1}{n} \text { for } \omega_{1}^{2}<\omega^{2}<n 。
$$

Consider again $n<\omega^{2}<\omega_{2}^{2}$ where $(n+2) \omega_{2}^{2}-\omega_{2}^{4}=n+2$. Then again $H\left(n \omega^{2}+2 \omega^{2}-\omega^{4}\right)$ is equal to 1 by Eq. (A7), and using Eq. (A5) we get

$$
H\left(\omega^{2}\right)=\frac{1}{2} \text {, for } n<\omega^{2}<\omega_{2}^{2} \text {. }
$$

Thus we have determined the function $H\left(\omega^{2}\right)$ in the interval $\omega_{1}^{2}<\omega^{2}<\omega_{2}^{2}$. This can be used to determine the function $H\left(\omega^{2}\right)$ in the intervals $\omega_{3}^{2}<\omega^{2}<\omega_{4}^{2}$ and $\omega_{5}^{2}<\omega^{2}$ $<\omega_{6}^{2}$ where $\omega_{3}^{2}, \omega_{4}^{2}, \omega_{5}^{2}$, and $\omega_{6}^{2}$ are constants determined by the equations

$$
\begin{aligned}
& (n+2) \omega_{3}^{2}-\omega_{3}^{4}=(n+2) \omega_{6}^{2}-\omega_{6}^{4}=\omega_{1}^{2}, \\
& (n+2) \omega_{4}^{2}-\omega_{4}^{4}=(n+2) \omega_{5}^{2}-\omega_{5}^{4}=\omega_{2}^{2},
\end{aligned}
$$

and so on. Eventually $H\left(\omega^{2}\right)$ is determined in all the interval $0<\omega^{2}<n+2$ except for some small set of zero measure. Thus we see that $H\left(\omega^{2}\right)$ is constant everywhere except for an infinite but denumerable number of points where its value increases discontinuously. Thus $D\left(\omega^{2}\right)$ which is the derivative of $H\left(\omega^{2}\right)$ and is the spectral density of the system is a sum of an infinite number of delta functions. Also the points $\omega^{2}=0$ and $\omega^{2}$ $=n+2$ are clearly the points of accumulation of the delta functions and hence again by Eqs. (A4) and (A5) there are an infinite number of such points of accumulation. Clearly the spectral density $D\left(\omega^{2}\right)$ is a highly singular function of $\omega^{2}$. However in thermodynamics we are usually interested only in integrals of $D\left(\omega^{2}\right)$ multiplied by a sufficiently smooth function of $\omega^{2}$. Hence only some sort of smeared value of $D\left(\omega^{2}\right)$ is of interest. It is easy to prove that in the case there exists nonzero finite positive constants $A$ and $B$ such that

$$
\begin{aligned}
& H\left(\omega^{2}\right) \leqslant A \omega^{d}, \quad \text { for } n+2 \geqslant \omega^{2} \geqslant 0, \\
& H\left(\omega^{2}\right) \geqslant B \omega^{d},
\end{aligned}
$$

where

$$
d=2 \ln (n) / \ln (n+2) .
$$

This result is sufficiently strong to permit us to identify $d$ as the effective dimensionality of the lattice.

\section{ACKNOWLEDGMENTS}

I am deeply indebted to my advisor, Professor Jon Mathews, for his encouragement, discussions, and critical comments throughout the course of this investigation. The financial support of the $H$. Dudley Wright Foundation is gratefully acknowledged.

*Richard P. Feynman Fellow.

${ }^{1}$ D.R. Nelson and M.E. Fisher, Ann. Phys. (N.Y.) 91, 226 (1975).

${ }^{2}$ K. Wilson and J. Kogut, Phys. Rep, C 12, 75 (1974).

${ }^{3}$ M.E. Fisher, Rep. Prog. Phys. 30, 615 (1967).

${ }^{4}$ K. Wilson, Phys. Rev, D 7, 2924 (1973).

${ }^{5}$ C.M. Fortuin and P.W. Kastelyn, Physica 57, 536 (1972).

${ }^{6}$ C.M. Fortuin, Physica 58, 393 (1972)

${ }^{7}$ C. M. Fortuin, Physica 59, 545 (1972). 